\title{
APLICAÇÃO DOS CUIDADOS COM OS PÉS ENTRE PORTADORES DE DIABETES MELLITUS
}

Renata Di Pietro Carvalho ${ }^{1}$, Carolina Di Pietro Carvalho ${ }^{1}$, Dulce Aparecida Martins ${ }^{2}$

RESUMO: Estudo descritivo realizado em uma unidade de saúde com Estratégia de Saúde da Família com o objetivo de identificar a aplicação de cuidados com os pés entre portadores de Diabetes Mellitus. Foram realizadas entrevistas no domicílio com aplicação de instrumento de coleta de dados e observação dos pés de 39 pacientes. Os resultados demonstraram que os portadores de diabetes apresentam falhas na aplicação das medidas preventivas do pé diabético, o que aumenta os riscos de complicações e incapacidades para as atividades cotidianas.

PALAVRAS-CHAVE: Diabetes mellitus; Pé diabético; Complicações do diabetes; Prevenção primária.

\section{FEET CARE AMONG DIABETES MELLITUS PATIENTS}

\begin{abstract}
It is a descriptive study developed in the Family Health Strategy unit, which aim is to identify the feet care among Diabetes Mellitus patients. Home interviews were conducted through the data collection instrument, as well as the observation of the feet of 39 patients. The results showed that individuals with diabetes have gaps in the knowledge when performing preventive measures for the diabetic foot, which increases the risk of complications and disability at daily ativities.
\end{abstract}

KEYWORDS: Diabetes mellitus; Diabetic foot; Diabetes complications; Primary prevention.

\section{APLICACIÓN DE LOS CUIDADOS DE LOS PIES ENTRE PORTADORES DE DIABETES MELLITUS}

RESUMEN: Estudio descriptivo realizado en una unidad de salud con Estrategia Salud de la Familia, con el objetivo de identificar la aplicación de cuidados de los pies entre portadores de Diabetes Mellitus. Se realizaron entrevistas en el domicilio con la aplicación de instrumento de recolección de datos y observación de los pies de 39 pacientes. Los resultados demostraron que los portadores de diabetes presentan fallas en la aplicación de las medidas preventivas del pie diabético, lo que aumenta los riesgos de complicaciones e incacapacidades para las actividades cotidianas.

PALABRAS CLAVE: Diabetes mellitus; Pie diabético; Complicaciones de la diabetes; Prevención primaria.

${ }^{1}$ Acadêmica de Enfermagem da Universidade Federal dos Vales do Jequitinhonha e Mucuri-UFVJM.

${ }^{2}$ Professora Assistente do Departamento de Enfermagem da UFVJM.

Autor correspondente:

Dulce Aparecida Martins

Universidade Federal dos Vales do Jequitinhonha e Mucuri

Rua da Glória, 187 - 39100-000 - Diamantina-MG, Brasil

Recebido: 22/08/09

E-mail: dulcediamanti@yahoo.com.br

Aprovado: 09/01/10

Cogitare Enferm 2010 Jan/Mar; 15(1):106-9 


\section{INTRODUÇÃO}

O Diabetes Mellitus (DM) é uma doença de grande expressão em todo o mundo. Na fase crônica tem como complicação o desenvolvimento do pé diabético que pode levar a amputações, que representam um dos mais devastadores problemas associados à doença, provocando grande impacto socioeconômico e perda da capacidade produtiva. Este agravo à saúde está entre as 10 maiores causas de óbito na maioria dos países, gerando mortes precoces e poderiam ser evitadas ${ }^{(1-2)}$. No Brasil, esta patologia apresenta prevalência de $7,6 \%$ a 12\% na faixa etária compreendida entre 30 e 69 anos, além de alto grau $(46,5 \%)$ de desconhecimento do diagnóstico ${ }^{(3)}$.

O pé diabético desenvolve-se na fase crônica da doença; amputação dos membros inferiores decorrem de gangrenas e/ou infecção severa. Tal situação evolui silenciosamente, o que requer do portador conhecimento para evitar este agravo ${ }^{(4)}$. As ulcerações nos pés decorrem de hábitos inadequados como andar descalço, uso de sapatos apertados, corte inadequado das unhas e pequenas dermatoses (micose, rachaduras, calos e deformidades dos pés). Esses fatores não representam grandes problemas e não causam maiores impactos às pessoas saudáveis, mas nos diabéticos podem ocasionar significativos danos.

O desenvolvimento do pé diabético e possível amputação dos membros inferiores é um problema físico e também psicológico, pois acarreta redução da autoestima, levando ao surgimento de outras doenças, como a depressão. A adoção de práticas simples de autocuidado com os pés e controle do diabetes contribui para a prevenção dos agravos, considerando-se que pacientes com melhor controle do DM são os que mais aplicam medidas preventivas do pé diabético ${ }^{(5)}$.

São também fatores coadjuvantes à ocorrência de úlceras nos pés a neuropatia periférica, a falta de informação sobre os cuidados com os pés, a presença de pontos de pressão anormal que favorecem calosidades, as deformidades, a doença vascular periférica e as dermatoses. Os pacientes com história prévia de úlceras ou amputações são considerados de elevado risco para desenvolvimento de novas úlceras e por isso necessitam maior atenção ${ }^{(6)}$.

A educação como medida preventiva deve ter o objetivo voltado para a motivação e a habilidade dos pacientes em reconhecer problemas e ações a serem adotadas. A diminuição das complicações depende de informações recebidas, sensibilização para mudanças no estilo de vida e desenvolvimento de habilidades para o autocuidado ${ }^{(1-4)}$, sendo relevante que também profissionais de saúde recebam educação para melhor atender e cuidar dos pacientes de alto risco.

A abordagem do pé diabético constitui um desafio em todo o mundo, principalmente nos países mais pobres, onde se enfrentam muitas dificuldades, como preconceitos e desconhecimento do assunto. A maioria dos diabéticos não é acompanhada em centros especializados, devido à falta de recursos e de pessoal adequadamente formado ${ }^{(4-7)}$.

A identificação dos níveis de conhecimento sobre autocuidado entre diabéticos e por parte das autoridades em saúde pode cooperar para o direcionamento de políticas voltadas à capacitação dos profissionais dessa área, contribuindo para amenizar o sofrimento e as complicações, diminuído os gastos com assistência especializada.

O enfermeiro desempenha uma função importante nos diversos níveis de atenção à saúde como agente cuidador e/ou educador, que na prática de prevenção acaba tendo maior importância ${ }^{(1)}$. Assim, é fundamental para o tratamento e prevenção das complicações que profissionais de saúde, clientes diabéticos e suas famílias tenham acesso a informações qualificadas.

O objetivo deste estudo consiste em identificar a aplicação de cuidados com os pés entre portadores de Diabetes Mellitus.

\section{METODOLOGIA}

Trata-se de um estudo descritivo realizado em uma Unidade de Saúde com Estratégia de Saúde da Família (ESF), numa cidade do interior de Minas Gerais; a população de estudo foi composta por 52 portadores de DM cadastrados nesta unidade. Foram considerados critérios de inclusão no estudo: ser portador de DM, não ter sido submetido a amputações, manter-se livre de grandes lesões por período igual ou superior a um ano (informações obtidas no prontuário dos pacientes cadastrados) e serem contatados por meio de visita domiciliar. Estas foram realizadas em horário comercial, junto com os agentes comunitários de saúde, durante período de agosto a novembro de 2008.

Os dados foram coletados durante a entrevista e com aplicação de instrumento com informações demográficas e um check list dos cuidados preventivos do pé diabético, além da observação dos pés buscando identificar os cuidados realizados pelos portadores de 
DM. Os dados foram submetidos à análise estatística.

Este trabalho de pesquisa foi aprovado pelo Comitê de Ética em Pesquisa da Universidade Federal dos Vales do Jequitinhonha e Mucuri sob parecer $\mathrm{n}^{0}$ 032/09 e desenvolvido de modo a garantir o cumprimento dos preceitos da Resolução 196/96 do Comitê Nacional de Ética em Pesquisa.

\section{ANÁLISE DOS RESULTADOS E DISCUSSÃO}

Participaram do estudo 39 pacientes com média de idade de 61,6 anos, sendo 32 do sexo feminino (82,05\%). Estudo realizado no Acre ${ }^{(5)}$ identificou média de idade de 59,2 com prevalência de mulheres (71,6\%). Quanto ao DM, 31(79,5\%) eram portadores do tipo II e $8(20,5 \%)$ do tipo I, resultados condizentes com pesquisas com predominância do DM tipo $2^{(1,10)}$. Este acomete indivíduos a partir dos 40 anos de vida, relacionado a hábitos de vida não saudáveis e sedentarismo. Essa tendência é mundial e reflete os "novos” hábitos de vida entre as populações, cada vez mais sedentárias.

O número oficial de $\mathrm{DM}$ associado à HAS é de 1.741.168, no período entre 1999 e 2002, no Brasil ${ }^{(10)}$. Caso não sejam feitos investimentos em ações educativas, esse problema poderá se tornar cada vez mais crítico, com amplo impacto sobre os custos no setor terciário de saúde, em função das complicações decorrentes.

Quanto à terapêutica, três $(7,69 \%)$ faziam uso somente de insulina, 26(66,6\%) utilizavam apenas hipoglicemiantes orais, três $(7,69 \%)$ faziam uso de hipoglicemiantes orais junto a insulina e sete $(17,94 \%)$ realizavam o controle do diabetes apenas com dieta. Encontramos na literatura que a insulina é utilizada por $37 \%$ dos sujeitos e o hipoglicemiante oral por $44 \%$ dos sujeitos portadores de $\mathrm{DM}^{(1)}$.

Em relação a patologias associadas, observouse que $27(69,23 \%)$ apresentam HAS, um(2,56\%) cardiopatia e 11(28,2\%) não apresentam patologia associada. Esse resultado está em conformidade com a literatura no que se refere à HAS; comorbidade comum nos pacientes diabéticos, representando risco adicional para o desenvolvimento de complicações macrovasculares e predispondo a amputações ${ }^{(1,5,9)}$.

O tempo de diagnóstico da doença foi variável: 14(35,89\%) tinham até cinco anos com o diagnóstico; 9 (23,07\%) entre cinco até 10 anos; quatro(10,25\%) com 10 a 15 anos; e quatro com diagnóstico há mais de 15 anos; 8(20,51\%) não souberam informar.
Ressalta-se que a precocidade do diagnóstico aliada, à adesão ao tratamento, é relevante na prevenção de agravos, como o pé diabético. $\mathrm{O}$ manejo insuficiente da doença favorece o aumento de complicações a longo prazo, aumentando risco e custo do tratamento. Assim, ao diagnosticar a doença, é imperativo trabalhar a questão da conscientização da clientela sobre a necessidade de boas práticas de saúde e autocuidado. Também é necessário que a clientela seja incentivada a participar do gerenciamento das políticas públicas de saúde, intervindo na aplicação dos recursos através de participação em conselhos de saúde e associações.

Quanto ao autocuidado, verificou-se que apenas $9(23,07 \%)$ realizavam todas as medidas preventivas: hidratação dos pés, limpeza dos pés com água morna e sabão neutro, exame diário dos pés e manutenção dos pés secos. Quinze(38,46\%) não realizavam medidas preventivas do pé diabético; quatro(10,25\%) faziam limpeza dos pés com água morna e sabão neutro, examinavam os pés diariamente e os mantinham secos; dois(5,12\%) hidratavam, secavam e examinavam os pés; dois(5,12\%) hidratavam e secavam os pés; dois(5,12\%) higienizavam e secavam os pés; dois(5,12\%) apenas higienizavam os pés; um(2,56\%) secava e examinava os pés; um(2,56\%) apenas examinava os pés e um (2,56\%) somente hidratava os pés. Na literatura, encontramos que $35 \%$ dos portadores de DM estudados sequer examinam os pés, o que indica uma preocupante falta de autocuidado voltado para a prevenção de complicações $^{(1)}$; esses apresentam alto risco para desenvolver o pé diabético.

Outro aspecto importante e que deve ser levado em consideração pelos portadores de DM é o cuidado inadequado com os pés e a escolha de calçados que favorecem o aparecimento de lesões, fatos intimamente relacionados ao desenvolvimento de deformidades, lesões e infecção, e predisponentes para o desenvolvimento do pé diabético e consequentes complicações $^{(1)}$.

Quinze (38,46\%) apresentavam corte das unhas e escolha dos calçados adequados, 12 (30,76\%) faziam de forma inadequada, ou seja, calçados que não protegem o suficiente contra lesões; $7(17,94 \%)$ mantinham calçado adequado e corte de unhas inadequado e cinco (12,82\%) faziam uso incorreto de calçados, com o corte de unhas correto. Sapato adequado é aquele que permite transpiração, oferece suporte às estruturas dos pés, tem bico que não aperta os dedos, é fechado e, se necessário, com palmilhas especiais. O corte das unhas adequado é aquele 
realizado após o banho ou colocação dos pés em água morna, favorecendo o amolecimento das unhas. O ângulo de corte deve ser reto e evitar cortar as unhas muito rentes ou remover as cutículas, a fim de evitar lesões. O calçado inadequado foi um dado importante, encontrado em $92 \%$ e o corte inadequado das unhas em $72 \%$ dos sujeitos investigados ${ }^{(1)}$.

Assim, aqueles que não desenvolvem nenhuma ou apenas algumas das medidas de autocuidado são considerados pacientes de risco para o desenvolvimento do pé diabético. Atenta-se para a necessidade de revisar a abordagem da clientela pelas equipes de saúde, com capacitação dessas, tendo em vista que a sensibilização para o problema pode mudar comportamentos. A educação dos pacientes pode contribuir para a mudança de comportamentos, fazendo com que melhore a adoção de práticas de autocuidado voltadas para a prevenção do pé diabético ${ }^{(5)}$. Tal prevenção é tarefa complexa que exige participação e responsabilidade por parte dos pacientes e dos profissionais para a identificação de problemas reais e potenciais, evitando assim o desenvolvimento de complicações.

\section{CONSIDERAÇÕES FINAIS}

Esse estudo demonstrou que os portadores do DM apresentam falhas na aplicação das medidas preventivas do pé diabético, podendo incorrer em aumento do risco de complicações e incapacidades, com prejuízos para a qualidade de vida. Considerando que a totalidade dos investigados não apresenta história de amputação ou lesões recentes nos pés, é relevante a intervenção junto a essa clientela com vistas à prevenção de agravos - como o pé diabético - com a orientação de pacientes e familiares sobre ações de prevenção e de autocuidado. Também é necessário investigar quais são as possíveis causas para a nãoadesão às práticas preventivas, como a falta de conhecimento.

O manejo dos pés de pessoas com diabetes é complexo e exige uma estreita colaboração e responsabilidade dos pacientes e dos profissionais, a fim de identificar problemas reais e potenciais, como estratégia para evitar o desenvolvimento de complicações.

\section{REFERÊNCIAS}

1. Laurindo MC, Recco DC, Robert DB, Rodrigues CDS. Conhecimento das pessoas diabéticas acerca dos cuidados com os pés. Arq Ciênc Saúde. [periódico na
Internet] Abr/Jun 2005;12(2): 80-84. [acesso em 2006 Nov 2] Disponível: http://www.cienciasdasaude.faemrp. br/racs_ol/Vol-12-2/4.pdf

2. Duran ECM, Cocco MIM. Software educativo sobre diabetes mellitus para profissionais de saúde: etapas de elaboração e desenvolvimento. Rev Latino-Am Enfermagem 2003 Jan/Fev;11(1):104-7.

3. Ministério da Saúde (BR). Secretaria de Políticas de Saúde. Departamento de Atenção Básica. Cadernos de Atenção Básica. Caderno 7. Hipertensão arterial sistêmica e diabetes mellitus. Protocolo. Brasília; 2001.

4. Fidelis C. Pé diabético. In: Pitta GBB, Castro AA, Burihan E, editores. Angiologia e cirurgia vascular: guia ilustrado. Maceió: UNCISA/ECMAL e LAVA, 2003. p. 11-21.

5. Cosson ICO, Ney-Oliveira F, Adan LF. Avaliação do conhecimento de medidas preventivas do pé diabético em pacientes do Rio Branco, Acre. Arq Bras Endocrinol Metabol. [periódico na Internet] 2005 Ago;49(4):548-56 [acesso em 2006 Nov 3]. Disponível: http://www.scielo. br/

6. Gross JL, Nehme M. Detecção e tratamento das complicações crônicas do diabetes melito: Consenso da Sociedade Brasileira de Diabetes e Conselho Brasileiro de Oftalmologia. RAMB. 1999;45(3):279-84.

7. Teixeira CRS, Zanetti ML. Serviço de medicina preventiva: atendimento multiprofissional em diabetes mellitus - uma possibilidade na prática em enfermagem. In: Telles Filho PCP, Gabbo AFF. A enfermagem na atualidade: ensino, pesquisa e extensão. $1^{\mathrm{a}} \mathrm{ed}$. São Paulo(SP):Gráfica São Paulo, 2003. p.72-92

8. Ochoa-Vigo K, Pace AE. Pé diabético: estratégias para prevenção. Acta Paul Enferm 2005 Jan/Mar;18(1):100-9.

9. Toscano CM. As campanhas nacionais para detecção das doenças crônicas não transmissíveis: diabetes e hipertensão arterial. Ciênc Saúde Coletiva [periódico na Internet] 2004 Out/Dez;9(4):885-95. [acesso em 2009 Out 10] Disponível em: http://www.scielo.br

10. Ministério da Saúde (BR). Sistema de cadastramento e acompanhamento de hipertensos e diabéticos: Hiperdia. 2002 [acesso em 2009 Out 9]. Disponível: http:// hiperdia.datasus.gov.br/ 